\section{Parto humanizado e tipo de parto: avaliação da assistência oferecida pelo Sistema Único de Saúde em uma cidade do Sul do Brasil}

Elizabeth Eriko Ishida Nagahama 1 Silvia Maria Santiago 2

\section{Humane childbirth and type of childbirth: an evaluation of care provided by the Brazilian National Health System in a city in the South of Brazil}

1 Hospital Universitário de Maringá. Universidade Estadual de Maringá. Av. Mandacaru, 1590. Parque das Laranjeiras. Maringá, PR, Brasil. CEP: 87.083-240.E-mail: eeinagahama@uem.br

2 Departamento de Medicina Preventiva e Social. Faculdade de Ciências Médicas. Universidade Estadual de Campinas. Campinas, SP, Brasil.

\section{Resumo}

Objetivos: avaliar a qualidade da atenção ao parto nos dois hospitais de referência para atendimento ao parto no Sistema Único de Saúde - SUS no município de Maringá, Paraná.

Métodos: estudo transversal, conduzido mediante pesquisa em prontuário hospitalar e entrevistas com 569 mulheres. Foram utilizados sete indicadores de processo, sendo quatro relacionados ao trabalho de parto e três ao parto. Por meio do estabelecimento de escores para cada um dos indicadores a assistência hospitalar foi qualificada em quatro niveis: excelente, boa, regular e insatisfatória. $O$ tipo de parto foi considerado indicador de resultado. A análise foi realizada nos softwares Excel e Statistica e utilizados os testes Qui-quadrado e T de Student.

Resultados: 1,0\% das mulheres tiveram atendimento excelente; $28,9 \%$ boa; $52,7 \%$ regular; e $17,4 \%$ insatisfatória. Na média geral de escores, o hospital 1 apresentou média superior ao do hospital 2.0 parto cesariana prevaleceu nos dois hospitais, com taxas superiores a $50 \%$.

Conclusões: os hospitais obtiveram desempenho classificado como regular e resultado da assistência insatisfatório, determinado pelas altas taxas de cesarianas.

Palavras-chave Saúde materno-infantil, Parto humanizado, Indicadores de qualidade em assistência à saúde 


\section{Introdução}

O número de mortes maternas de um país constitui um bom indicador de sua condição social e da qualidade da assistência ao pré-natal, parto, puerpério e aborto. Seus níveis servem como uma forma de estimar possíveis iniquidades, na medida em que revelam grandes disparidades, ao se compararem áreas ou regiões em diferentes graus de desenvolvimento. ${ }^{1}$

Nos países desenvolvidos, a média de sete mortes maternas, com variação de quatro a quatorze, para cada 100 mil nascidos vivos, contrasta com as registradas no Brasil, que contabilizou, no ano de 2007, 77 mortes para cada 100 mil nascimentos. Esta taxa posiciona o país como $29^{\circ}$ colocado nos números absolutos mais altos de mortes maternas. No Brasil, uma em cada 370 brasileiras corre o risco de morrer devido a complicações na gravidez ou no parto.1,2 As síndromes hipertensivas, as hemorragias, as infecções puerperais e as complicações do aborto são responsáveis por $66 \%$ das mortes maternas no Brasil. ${ }^{3}$

A taxa abusiva de parto cesáreo no país desde a década de 1980 constitui um motivo de complicações no parto e é um dos principais exemplos do modelo assistencial excessivamente intervencionista. O parto cesáreo representa $80 \%$ dos partos realizados via planos privados de saúde e $26 \%$ no sistema público, o que equivale a uma taxa média de cesarianas de $40 \% .4$ Esta situação coloca o país como líder mundial em freqüência de cesáreas. 4

Cada cesariana desnecessária significa um risco maior de complicações, como infecção, hemorragia e complicações anestésicas, as quais contribuem com o aumento das taxas de mortalidade materna. Para a criança, o risco principal refere-se aos problemas respiratórios advindos da prematuridade, geralmente associados com a retirada antecipada do concepto antes da maturidade pulmonar plena. Além disso, o excesso de procedimentos cirúrgicos, internações e tratamentos de complicações representam o desperdício de milhões de reais por ano no Sistema Único de Saúde (SUS). Estudos mostram que o parto cesariano custa, em geral, de duas a três vezes o valor do parto normal. 5

Assim, grande parte das mortes maternas poderia ser evitada mediante a redução das taxas de cesarianas, com a melhoria na qualidade da atenção obstétrica nos serviços de saúde, no incentivo ao parto vaginal e, principalmente, com a implantação do parto humanizado nos serviços de saúde.

No final da década de 1990, o tema da humanização na atenção foi incorporado aos programas de saúde propostos pelo Ministério da Saúde do Brasil. No ano de 2003, destacou-se a implantação da Política Nacional de Humanização, na qual humanizar a atenção e a gestão em saúde no SUS constituiu ferramenta para qualificar as práticas de saúde. ${ }^{6} \mathrm{O}$ conceito de parto humanizado é amplo, polissêmico e pode ser explorado em várias dimensões e de formas complementares entre si. ${ }^{7,8}$ Adotouse para esse estudo o conceito da World Health Organization (WHO), ${ }^{9}$ qual seja: "um conjunto de condutas e procedimentos que visam à promoção do parto e do nascimento saudável e à prevenção da morbimortalidade perinatal. Incluem o respeito ao processo fisiológico e à dinâmica de cada nascimento, nos quais as intervenções devem ser cuidadosas, evitando-se os excessos e utilizando-se, criteriosamente, os recursos tecnológicos disponíveis".

O parto humanizado compreende o atendimento centrado na mulher, individualizado, fundamentado na medicina baseada em evidências, no respeito à evolução fisiológica do parto e, portanto, na indicação criteriosa dos partos cesáreos, que não deve ultrapassar a taxa de $15 \%$, conforme recomenda a WHO. 10

Em 1996, a WHO9 publicou um guia prático com as recomendações para implantação do parto humanizado nos serviços de saúde. As rotinas de assistência ao parto e nascimento propostas são classificadas em quatro categorias. Na primeira, descreve práticas que deveriam ser estimuladas, em virtude da sua utilidade e respeito à parturiente; na segunda, estão aquelas que deveriam ser eliminadas, por seu caráter danoso ou ineficaz; na terceira, mostra condutas sem evidência suficiente para apoiar uma recomendação e que deveriam ser usadas com precaução; e, na última, lista procedimentos usados regularmente, porém de maneira inapropriada.

A primeira categoria, denominada de categoria A, ${ }^{9}$ enumera vinte e duas práticas classificadas como claramente úteis e que devem ser utilizadas na atenção obstétrica. Baseado nesta categoria, o Ministério da Saúde do Brasil implantou, no ano 2000, o "Programa de Humanização no Pré-natal e Nascimento", 11 que objetiva ampliar o acesso das mulheres aos serviços de saúde, humanizar a assistência, garantir a qualidade da atenção, incentivar o parto vaginal e, desta forma, reduzir os coeficientes de mortalidade materna no país. O programa repassa incentivos financeiros a municípios e maternidades que ofereçam, em sua rede de serviços, atenção sistematizada em protocolos e de qualidade no pré-natal, parto e puerpério.

O município de Maringá está localizado no Estado do Paraná, região Sul do Brasil e contabiliza 
cerca de 325.000 habitantes. No município, dois hospitais gerais um público e, o outro beneficente constituem referência para partos na rede pública de saúde. O modelo assistencial excessivamente intervencionista na assistência ao parto, expresso na alta taxa de cesáreas do município nos últimos anos (acima de 70\%),12 motivou a realização deste estudo, que objetivou avaliar a qualidade da atenção ao parto no município de Maringá Estado do Paraná, sob dois aspectos: o primeiro refere-se à avaliação do processo da qualidade da atenção, baseado no conceito de parto humanizado da $\mathrm{WHO}^{9}$ e o segundo consiste na avaliação do resultado da atenção prestada pelo serviço de saúde tendo como desfecho o tipo de parto.

\section{Métodos}

Realizou-se um estudo com desenho transversal no período de março/2005 a fevereiro/2006. Trata-se de estudo avaliativo conduzido por avaliadores internos e externos 13 aos serviços de saúde.

A população de estudo foi constituída por mulheres que tiveram o parto vaginal ou cesáreo nos dois hospitais de referência para parto no SUS (nomeados de hospital 1 e 2), no município de Maringá, Estado do Paraná, Brasil.

O hospital 1 caracteriza-se como sendo de caráter público, hospital de ensino, Hospital Amigo da Criança e constitui referência no atendimento a gestantes de alto risco para 30 municípios da macroregião noroeste do Paraná. O hospital 2, credenciado pelo SUS, é um hospital geral, beneficente, sem fins lucrativos e é referência para atendimento hospitalar ao parto para gestantes de baixo e alto risco atendidas em 19 unidades básicas de saúde do município. O tamanho da amostra foi estimado com base no número de nascidos vivos dos dois hospitais, no período de março/2004 a fevereiro/2005. A amostra foi calculada com $95 \%$ de confiança e erro máximo de $5 \%$. Estimou-se uma amostra para cada hospital, estratificada por tipo de parto e mês, que totalizou 569 mulheres, sendo 259 no hospital 1 (136 partos cesáreos e 123 partos vaginais) e 310 no hospital 2 (160 partos cesáreos e 150 partos vaginais).

No período de 01/03/2005 a 28/02/2006 nasceram 2290 crianças vivas nos dois hospitais de referência para atendimento ao parto no SUS no município de Maringá. Dentre estas, 744 (32,5\%) ocorreram no hospital 1 e 1546 (67,5\%) no hospital 2. A amostra, portanto, cobriu $34,8 \%$ dos partos no hospital 1 e $20,1 \%$ no hospital 2.

Para a coleta de dados foram realizadas entrevistas por meio de questionário estruturado consti- tuído de duas partes: a primeira objetivou a transcrição de informações demográficas, sociais e história reprodutiva constantes no prontuário hospitalar. A segunda englobou questões que indicaram a existência de práticas humanizadas na assistência no trabalho de parto e durante o parto.

As entrevistadoras receberam uma planilha mensal, na qual constou o número de mulheres a serem entrevistadas por tipo de parto e o roteiro dos leitos sorteados. Baseado no número de leitos de puerpério disponíveis em cada hospital (seis no hospital 1 e 15 no hospital 2) e com auxílio do programa excel foram construídos seis combinações possíveis de roteiros de leitos para entrevista em cada hospital. Os roteiros, que objetivaram aleatorizar a amostra, foram incluídos na planilha mensal e indicavam a sequência dos leitos a ser seguida pelo entrevistador para a realização da entrevista.

Cinco entrevistadoras aplicaram um questionário entre o primeiro e terceiro dia de puerpério, no alojamento conjunto de cada hospital, após o consentimento da mulher ou responsável.

Trinta e sete mulheres não foram entrevistadas pelos seguintes motivos: ocorrência do parto fora do ambiente hospitalar (2), óbito fetal (3), recusa em participar da pesquisa (9) e adolescente menor de idade (menos que 18 anos) sem acompanhante que autorizasse a realização da entrevista (23). Estas mulheres foram substituídas pela subsequente, conforme indicação do roteiro de leito para entrevista em cada hospital.

Para a avaliação do processo da qualidade da atenção, foram utilizados sete indicadores vinculados à humanização, sendo quatro relacionados ao trabalho de parto e três ao parto. A seleção destes indicadores foi baseada na Categoria A da WHO. ${ }^{9}$

Os indicadores de qualidade na assistência ao trabalho de parto e no parto estão listados a seguir:

\section{Durante o trabalho de parto}

a) Fornecimento às mulheres de todas as informações e explicações que desejassem (indicador 1): representa o empoderamento das mulheres, indica a atenção pessoal e individualizada e o apoio do profissional de saúde no atendimento à mulher; 3

b) Presença do acompanhante durante o trabalho de parto (indicador 2): a presença do acompanhante no trabalho de parto é associado à redução da duração do trabalho de parto, redução da administração de ocitocina na indução do trabalho de parto, diminuição da taxa de cesariana, do uso do fórcipe e da necessidade de analgesia; 3,14

c) Uso de métodos não-invasivos e não-farma- 
cológicos de alívio da dor (indicador 3): apresentam alto grau de segurança e simplicidade na sua utilização; seu uso associa-se à menor necessidade de sedativos e de analgesia peridural, redução do tempo do trabalho de parto, maior proporção de partos vaginais e satisfação da mulher com o parto. Além disso, representam a valorização da dor da parturiente e apontam para a atenção individualizada do profissional de saúde; 15,16

d) Oferecimento de líquidos por via oral (indicador 4): previne a desidratação e a acidose. ${ }^{9}$

\section{Durante o parto}

e) Fornecimento às mulheres de todas as informações e explicações que desejassem no parto (indicador 5): representam o apoio pessoal e atenção individualizada à mulher; 3

f) Presença do acompanhante no parto (indicador 6): o acompanhante evita o isolamento e a violência institucional e constitui reflexo da mudança de atitude institucional, em particular, dos profissionais de saúde;3,14

g) Promoção do contato pele-a-pele entre mãe e filho em sala de parto (indicador 7): abrange benefícios nos aspectos psico-afetivos, orgânicos e fisiológicos. Promove a interação precoce entre mãe-filho, formação de vínculo, aleitamento materno exclusivo por período mais prolongado e amamentação quantitativamente melhor.9,15

Para a avaliação do resultado da atenção prestada pelo serviço de saúde, foi selecionado um único indicador de resultado: tipo de parto. Ao considerar-se que $o$ atendimento humanizado qualifica a assistência e contribui para a evolução fisiológica do parto, ${ }^{9}$ o tipo de parto foi considerado indicador da efetividade do cuidado.

O tipo de parto foi verificado por meio do registro de número de nascidos vivos obtidos nas Declarações de Nascidos Vivos (DNV) de crianças nascidas nos dois hospitais, no período do estudo. Adotou-se como parâmetro para este indicador de resultado o percentual máximo de $35 \%$ de cesarianas, estabelecido para o Estado do Paraná no ano de 2000.17

Para estabelecer os critérios de avaliação da qualidade na assistência ao parto, utilizou-se escala de escores, com variação de 0 a 4 pontos, de acordo com o cumprimento de cada um dos critérios. Para os indicadores 1 e 5 , a resposta "sim" equivaleu a 2 pontos, "em parte" a 1 ponto e "não" a zero; em relação ao indicador 2 , o escore foi atribuído conforme o tempo de permanência do acompanhante no trabalho de parto: se mais da metade do tempo do trabalho de parto: 2 pontos, se metade do tempo de trabalho de parto ou menos: 1 ponto e a ausência do acompanhamento equivaleu a zero; o escore total do indicador três (uso de métodos não farmacológicos de alívio da dor) foi obtido mediante a soma dos escores de cada item: o uso dos quatro métodos: massagens, banho de chuveiro, música e exercícios de respiração equivaleu a 4 pontos; o uso de três métodos correspondeu a 3 pontos; o uso de dois métodos recebeu escore de 2 pontos; o uso de um dos métodos, 1 ponto e a ausência de utilização de método equivaleu a zero. Considerou-se maior pontuação para este indicador por representar a adesão ao ideário da humanização e o investimento do profissional de saúde na evolução fisiológica do trabalho de parto e no favorecimento do parto vaginal. Quanto ao oferecimento de líquidos por via oral durante o trabalho de parto (indicador 4), se positivo equivaleu a 2 pontos e, se negativo, a zero ponto. A presença do acompanhante no parto (indicador 6) equivaleu a 2 pontos e a ausência correspondeu a zero ponto. $\mathrm{O}$ escore para o indicador 7 foi atribuído conforme o tempo de contato entre mãe e filho: se trinta minutos ou mais: 2 pontos, se menos que trinta minutos: 1 ponto e a ausência do contato pele a pele entre mãe e filho equivaleu a zero. A qualidade da assistência ao parto foi mensurada mediante a somatória de pontos obtidos em cada um dos sete indicadores, que permitiu qualificá-la num escore que variou de zero a dezesseis pontos. Pressupôs-se que a qualidade do cuidado poderia variar de insatisfatório a excelente, conforme os quatro níveis de qualidade: a) Insatisfatória: 0 a 4 pontos; b) Regular: 5 a 8 pontos; c) Boa: 9 a 12 pontos; d) Excelente: 13 a 16 pontos.

A análise dos dados foi realizada por meio dos programas MS Excel e Statistica 6.0. Para análise dos resultados, as associações entre duas variáveis (tipo de parto e hora de ocorrência; tipo de parto e qualidade da assistência; procedência da mulher e presença do acompanhante no trabalho de parto; história reprodutiva e ocorrência do parto vaginal; antecedentes de partos cesáreos e uso de métodos não farmacológicos de alívio da dor; antecedentes de partos cesáreos e oferecimento de líquidos via oral; escore médio por indicador de qualidade e tipo de parto) foram realizadas pelo teste qui-quadrado. Para comparação de médias, utilizou-se o teste $\mathrm{t}$ de Student. O nível de significância adotado foi de $5 \%$.

A pesquisa foi aprovada pelo Comitê de Ética em Pesquisa com Seres Humanos da Faculdade de Ciências Médicas da Universidade Estadual de Campinas, SP, Brasil, processo número 591/2004. 


\section{Resultados}

As mulheres entrevistadas tinham a média de 25 anos $(\mathrm{DP}=6,4)$, variando de 12 a 46 anos. Nas faixas etárias extremas do período reprodutivo, $24,3 \%$ eram adolescentes (10 a 19 anos de idade) e 6,7\% das mulheres tinham mais de 35 anos. Grande parte das mulheres $(74,8 \%)$ convivia com parceiro, $23,7 \%$ eram solteiras, $1,1 \%$ divorciadas/desquitadas e $0,4 \%$ viúvas. Quanto à escolaridade, $0,9 \%$ das mulheres eram analfabetas; $28,8 \%$ frequentou o primeiro grau; $65,9 \%$ cursou o segundo grau; e 4,4\% estudou até o nível superior. Na distribuição das mulheres quanto à procedência, verificou-se que grande parte $(76,4 \%)$ residia no município de Maringá, PR.

No que se refere à inserção no mercado de trabalho, $62,9 \%$ das mulheres não exerciam atividade remunerada. Mais da metade das mulheres que não exercia atividade remunerada era dona de casa $(58,5 \%)$ e $4,4 \%$ eram estudantes.
Em relação à história reprodutiva, $39,9 \%$ das mulheres eram primíparas e $60,1 \%$ multíparas, sendo $46,4 \%$ com um filho vivo; $31,3 \%$ dois e $22,3 \%$ tinham três ou mais filhos vivos. Registrou-se o percentual de $24,0 \%$ de mulheres com antecedentes de duas cesáreas ou mais. Encontrou-se 16,3\% das mulheres com antecedentes de abortamentos.

Conforme apresentado na Tabela 1, a maioria das mulheres $(81,5 \%)$ teve o parto a termo, $16,7 \%$ corresponderam à condição de pré-termo e $1,8 \%$ foram classificadas como pós-termo. A época da ocorrência do parto variou de 25 a 42 semanas, com a média de 39,1 (DP 1,08). semanas de gestação.

Observou-se associação entre a hora de ocorrência e tipo de parto $(p<0,001)$. O parto vaginal ocorreu com maior frequência no período noturno, sobretudo no turno compreendido entre 1:00-7:00 horas. No hospital 1 o parto cesáreo foi realizado com maior frequência no turno das 7:00-13:00 horas.

\section{Tabela 1}

Distribuição absoluta e percentual das características do atendimento hospitalar no trabalho de parto e parto em dois hospitais vinculados ao Sistema Único de Saúde. Maringá-PR, março/2005 a fevereiro/2006.

\begin{tabular}{|c|c|c|c|c|c|c|}
\hline \multirow[t]{2}{*}{ Variáveis } & \multicolumn{2}{|c|}{ Hospital 1} & \multicolumn{2}{|c|}{ Hospital 2} & \multicolumn{2}{|c|}{ Total } \\
\hline & $\mathrm{n}$ & $\%$ & $n$ & $\%$ & $n$ & $\%$ \\
\hline \multicolumn{7}{|l|}{ Idade gestacional (semanas) } \\
\hline Menos de 37 & 62 & 23,9 & 33 & 10,7 & 95 & 16,7 \\
\hline De 37 a 42 incompletas & 193 & 74,5 & 271 & 87,4 & 464 & 81,5 \\
\hline 42 e mais & 4 & 1,6 & 6 & 1,9 & 10 & 1,8 \\
\hline \multicolumn{7}{|l|}{ Tipo de parto } \\
\hline Vaginal sem fórcipe & 120 & 46,3 & 150 & 48,4 & 270 & 47,5 \\
\hline Vaginal com fórcipe & 3 & 1,2 & - & - & 3 & 0,5 \\
\hline Cesárea & 136 & 52,5 & 160 & 51,6 & 296 & 52,0 \\
\hline \multicolumn{7}{|l|}{ Ocorrência do parto (horas) } \\
\hline 1 às 7 & 37 & 14,3 & 45 & 14,6 & 82 & 14,4 \\
\hline 7 às 13 & 60 & 23,2 & 82 & 26,5 & 142 & 25,0 \\
\hline 13 às 19 & 72 & 27,8 & 82 & 26,5 & 154 & 27,0 \\
\hline 19 às 1 & 90 & 34,7 & 101 & 32,6 & 191 & 33,6 \\
\hline \multicolumn{7}{|c|}{ Local assistência no trabalhode parto } \\
\hline Pronto socorro & 149 & 57,5 & 4 & 1,3 & 153 & 26,9 \\
\hline Enfermaria & 109 & 42,1 & 306 & 98,7 & 415 & 72,9 \\
\hline Ambulatório & 1 & 0,4 & - & - & 1 & 0,2 \\
\hline \multicolumn{7}{|l|}{ Local realização do parto } \\
\hline Pronto socorro & 2 & 0,8 & - & - & 2 & 0,4 \\
\hline Enfermaria & 5 & 1,9 & 5 & 1,6 & 10 & 1,8 \\
\hline Centro cirúrgico & 252 & 97,3 & 305 & 98,4 & 557 & 97,8 \\
\hline \multicolumn{7}{|l|}{ Apgar quinto minuto } \\
\hline $4-6$ & 1 & 0,4 & 2 & 0,6 & 3 & 0,5 \\
\hline $7-10$ & 258 & 99,6 & 308 & 99,4 & 566 & 99,5 \\
\hline Total & 259 & 45,5 & 310 & 55,5 & 569 & 100,0 \\
\hline
\end{tabular}


Quanto ao local de realização dos partos nos hospitais estudados, 557 (97,8\%) partos foram realizados no centro cirúrgico.

A maioria dos recém-nascidos $(99,5 \%)$ obteve escore de Apgar entre 7 a 10 no quinto minuto de vida.

Verifica-se, na Tabela 2, que mais da metade das mulheres $(66,6 \%)$ referiu ter recebido as informações desejadas no trabalho de parto, 30,8\% manifestaram insatisfação com as informações recebidas e $2,6 \%$ estavam parcialmente satisfeitas. $\mathrm{Na}$ assistência em sala de parto, $77,1 \%$ das mulheres do hospital 2 receberam as informações desejadas, enquanto que no hospital 1 esta porcentagem foi de $58,7 \%$

Observou-se que estes dois indicadores ( 1 e 5) foram os que apresentaram melhores resultados na média geral de pontuações.

Foi permitida a presença do acompanhante no trabalho de parto (indicador 2) para pouco mais da metade das mulheres (58\%). Contudo, ao observar este indicador separadamente em cada hospital,

\section{Tabela 2}

Distribuição absoluta e percentual das características do atendimento hospitalar no trabalho de parto e parto em dois hospitais vinculados ao Sistema Único de Saúde. Maringá-PR, março/2005 a fevereiro/2006.

\begin{tabular}{|c|c|c|c|c|c|c|}
\hline \multirow[t]{2}{*}{ Variáveis } & \multicolumn{2}{|c|}{ Hospital 1} & \multicolumn{2}{|c|}{ Hospital 2} & \multicolumn{2}{|c|}{ Total } \\
\hline & $\mathrm{n}$ & $\%$ & $\mathrm{n}$ & $\%$ & $\mathrm{n}$ & $\%$ \\
\hline \multicolumn{7}{|l|}{ Trabalho de parto } \\
\hline \multicolumn{7}{|l|}{ 1. Recebeu informações } \\
\hline Sim & 156 & 60,2 & 223 & 71,9 & 379 & 66,6 \\
\hline Não & 98 & 37,8 & 77 & 24,9 & 175 & 30,8 \\
\hline Em parte & 5 & 1,9 & 10 & 3,2 & 15 & 2,6 \\
\hline \multicolumn{7}{|l|}{ 2. Acompanhante } \\
\hline Mais da metade do tempo & 164 & 63,3 & 115 & 37,1 & 279 & 49,0 \\
\hline Metade do tempo/ menos & 38 & 14,7 & 14 & 4,5 & 52 & 9,0 \\
\hline Não ficou & 57 & 22,0 & 181 & 58,4 & 238 & 42,0 \\
\hline \multicolumn{7}{|c|}{$\begin{array}{l}\text { 3. Métodos não farmacológicos de } \\
\text { alivio da dor }\end{array}$} \\
\hline Massagem & 73 & 28,2 & 46 & 14,8 & 119 & 20,9 \\
\hline Banho & 126 & 48,6 & 148 & 47,7 & 274 & 48,2 \\
\hline Música & 2 & 0,8 & 7 & 2,3 & 9 & 1,6 \\
\hline Respiração & 136 & 52,5 & 138 & 44,5 & 274 & 48,2 \\
\hline \multicolumn{7}{|l|}{ 4. Líquidos via oral } \\
\hline $\operatorname{Sim}$ & 130 & 50,2 & 137 & 44,2 & 267 & 46,9 \\
\hline Não & 129 & 49,8 & 173 & 55,8 & 302 & 53,1 \\
\hline \multicolumn{7}{|l|}{ Parto } \\
\hline \multicolumn{7}{|l|}{ 5. Recebeu informações } \\
\hline $\operatorname{Sim}$ & 152 & 58,7 & 239 & 77,1 & 391 & 68,7 \\
\hline Não & 97 & 37,4 & 60 & 19,4 & 157 & 27,6 \\
\hline Em parte & 10 & 3,9 & 11 & 3,5 & 21 & 3,7 \\
\hline \multicolumn{7}{|l|}{ 6. Acompanhante } \\
\hline Sim & 7 & 2,7 & 1 & 0,3 & 8 & 1,4 \\
\hline Não & 252 & 97,3 & 309 & 99,7 & 561 & 98,6 \\
\hline \multicolumn{7}{|l|}{ 7. Contato pele a pele } \\
\hline Trinta minutos ou mais & 39 & 15,1 & 1 & 0,3 & 40 & 7,0 \\
\hline Menos que trinta minutos & 177 & 68,3 & 283 & 91,3 & 460 & 80,8 \\
\hline Não & 43 & 16,6 & 26 & 8,4 & 69 & 12,2 \\
\hline Total & 259 & 45,5 & 310 & 55,5 & 569 & 100,0 \\
\hline
\end{tabular}


registra-se que $78 \%$ das mulheres tiveram acompanhante no hospital 1 , em contraste com o percentual de $41,6 \%$ encontrado no hospital $2(p<0,001)$.

Apenas oito $(1,4 \%)$ mulheres tiveram a presença do acompanhante na sala de parto (indicador 6) e, nos dois hospitais, registraram-se percentuais muito baixos: no hospital 1 foi de $2,7 \%$ e, no outro, de $0,3 \%$. Este indicador foi o que apresentou menor média de escores, ou seja, pior avaliação dentre os indicadores para os dois hospitais (Tabela 3).

Conforme a Tabela 3 pode-se observar que em relação ao uso de métodos não farmacológicos para alívio da dor no trabalho de parto (indicador 3), registrou-se que $383(67,3 \%)$ mulheres receberam um dos quatro métodos não-farmacológicos. Os mais utilizados foram o banho de chuveiro e os exercícios respiratórios $(48,2 \%)$. A massagem foi pouco utilizada $(20,9 \%)$, bem como a musicoterapia

\section{Tabela 3}

Média e desvio-padrão de escore por indicador da qualidade da atenção no trabalho de parto e parto em dois hospitais vinculados ao Sistema Único de Saúde. Maringá-PR, março/2005 a fevereiro/2006.

\begin{tabular}{|c|c|c|c|}
\hline \multirow{2}{*}{ Indicador } & Hospital 1 & Hospital 2 & \multirow{2}{*}{$p$} \\
\hline & $\bar{x} \pm D P$ & $\bar{x} \pm D P$ & \\
\hline 1. Informações no trabalho de parto & $1,22 \pm 0,97$ & $1,47 \pm 0,87$ & 0,001 \\
\hline 2. Acompanhante no trabalho de parto & $1,41 \pm 0,83$ & $0,79 \pm 0,96$ & $<0,001$ \\
\hline 3. Uso de métodos não-farmacológicos & $1,30 \pm 1,06$ & $1,09 \pm 1,03$ & 0,015 \\
\hline 4. Líquidos via oral no trabalho de parto & $1,00 \pm 1,00$ & $0,88 \pm 0,99$ & 0,154 \\
\hline 5. Informações no parto & $1,21 \pm 0,96$ & $1,58 \pm 0,80$ & $<0,001$ \\
\hline 6. Acompanhante na sala de parto & $0,05 \pm 0,32$ & $0,00 \pm 0,11$ & 0,016 \\
\hline 7. Contato pele-a-pele em sala de parto & $0,98 \pm 0,56$ & $0,92 \pm 0,28$ & 0,075 \\
\hline Avaliação geral (Escore) & $7,2 \pm 2,77$ & $6,7 \pm 2,48$ & 0,037 \\
\hline
\end{tabular}

$(1,6 \%)$. Na avaliação comparativa da média de escores para este indicador, o hospital 1 apresentou melhor resultado que o 2 . No que se refere ao indicador 4 , registrou-se que $46,9 \%$ das mulheres receberam líquidos no trabalho de parto. Na avaliação comparativa entre os hospitais (Tabela 3), ambos apresentaram médias de escore semelhantes para este item. Conforme se observa na Tabela 2, a maioria dos recém-nascidos $(87,8 \%)$ obteve o contato pele-a-pele com sua mãe na sala de parto (indicador 7). Apesar de o hospital 1 apresentar o percentual de contato pele-a-pele por trinta minutos ou mais superior ao 2, os hospitais apresentam médias de escore semelhantes neste item. Ressaltase que os indicadores 6 e 7 foram os que apresentaram pior avaliação nos hospitais. Ao comparar-se a média de escore de qualidade obtida nos dois hospitais, verificou-se que o hospital 1 obteve melhor resultado que o 2. O hospital 1 apresentou escore de $7,2(\mathrm{DP}=2,77)$ e, o segundo, escore de $6,7(\mathrm{DP}=$ $2,48)$, com diferença estatisticamente significativa $(p=0,03)$.

$\mathrm{Na}$ análise dos indicadores de qualidade por tipo de parto registrou-se associação entre parto vaginal e os indicadores: uso de métodos não farmacológicos de alívio da dor $(p<0,001)$, receber líquidos via oral no trabalho de parto $(p<0,001)$, presença do acompanhante na sala de parto $(p=0,024)$ e contato pelea-pele em sala de parto $(p=0,004)$. Por outro lado, evidenciou-se que as mulheres submetidas ao parto cesáreo apresentaram média de escore superior para o indicador receber informações que desejava na sala de parto $(p=0,017)$. Não houve associação significativamente estatística para os indicadores receber informações que desejava no trabalho de parto e presença do acompanhante no trabalho de parto (Tabela 4).

Outra informação relevante refere-se à média de escore de qualidade registrada para as mulheres submetidas ao parto vaginal e cesáreo. Verificou-se que a média de pontuação das mulheres que evoluíram para o parto vaginal foi superior $(\overline{\mathrm{X}}=7,5$; $\mathrm{DP}=2,77)$ às mulheres que foram submetidas ao parto cesáreo $(\overline{\mathrm{X}}=6,3 ; \mathrm{DP}=2,36)$, com diferença estatisticamente significativa $(p<0,001)$ (Tabela 4$)$. 
Média e desvio-padrão de escore por indicador da qualidade da atenção no trabalho de parto e parto por tipo de parto. Maringá-PR, março/2005 a fevereiro/2006.

\begin{tabular}{|c|c|c|c|}
\hline \multirow{2}{*}{ Indicador } & Parto vaginal & Parto cesário & \multirow{2}{*}{$p$} \\
\hline & $\bar{X} \pm D P$ & $\bar{X} \pm D P$ & \\
\hline 1. Informações no trabalho de parto & $1,34 \pm 0,93$ & $1,37 \pm 0,91$ & 0,657 \\
\hline 2. Acompanhante no trabalho de parto & $1,11 \pm 0,95$ & $1,04 \pm 0,95$ & 0,411 \\
\hline 3. Uso de métodos não-farmacológicos & $1,55 \pm 1,05$ & $0,85 \pm 0,93$ & $<0,001$ \\
\hline 4. Líquidos via oral no trabalho de parto & $1,20 \pm 0,98$ & $0,70 \pm 0,95$ & $<0,001$ \\
\hline 5. Informações no parto & $1,32 \pm 0,93$ & $1,50 \pm 0,85$ & 0,017 \\
\hline 6. Acompanhante na sala de parto & $0,05 \pm 0,32$ & $0,01 \pm 0,12$ & 0,024 \\
\hline 7. Contato pele-a-pele em sala de parto & $1,00 \pm 0,47$ & $0,90 \pm 0,40$ & 0,004 \\
\hline Avaliação geral (Escore) & $7,57 \pm 2,77$ & $6,36 \pm 2,36$ & $<0,001$ \\
\hline
\end{tabular}

Na classificação geral, segundo os indicadores escolhidos para a avaliação da qualidade da assistência ao parto, o escore final mostrou que $1,0 \%$ das mulheres tiveram atendimento excelente, $28,9 \%$ boa, $52,7 \%$ regular e $17,4 \%$ insatisfatória (Tabela 5).

É importante ressaltar a associação entre tipo de parto e qualidade da atenção $(p<0,001)$. Registrou-se frequência superior de ocorrência de partos vaginais entre os atendimentos categorizados como bom e excelente.

O tipo de parto que prevaleceu nos dois hospitais foi a cesariana, com o percentual de $51 \%$ no hospital 1 e de $52 \%$ no hospital 2. Portanto, o resultado da assistência foi considerado insatisfatório, pois a taxa de cesariana foi superior ao parâmetro de $35 \%$ estabelecido neste estudo.

Registrou-se associação entre antecedentes de partos vaginais e cesáreos e a ocorrência do parto

Tabela 5

Distribuição absoluta e percentual da classificação da qualidade da assistência ao parto em dois hospitais vinculados ao Sistema Único de Saúde. Maringá-PR, março/2005 a fevereiro/2006.

\begin{tabular}{|c|c|c|c|c|c|c|}
\hline \multirow{2}{*}{ Qualidade da assistência ao parto } & \multicolumn{2}{|c|}{ Hospital 1} & \multicolumn{2}{|c|}{ Hospital 2} & \multicolumn{2}{|c|}{ Total } \\
\hline & $\mathrm{n}$ & $\%$ & $\mathrm{n}$ & $\%$ & $n$ & $\%$ \\
\hline Excelente & 5 & 1,9 & 1 & 0,3 & 6 & 1,0 \\
\hline Boa & 84 & 32,4 & 80 & 25,8 & 164 & 28,9 \\
\hline Regular & 124 & 47,9 & 176 & 56,8 & 300 & 52,7 \\
\hline Insatisfatória & 46 & 17,8 & 53 & 17,1 & 99 & 17,4 \\
\hline Total & 259 & 45,5 & 310 & 55,5 & 569 & 100,0 \\
\hline
\end{tabular}

Teste exato de Fisher, $p=0.05$.

vaginal $(p<0,001)$ : mulheres com antecedentes de um a três partos vaginais e sem antecedentes de cesáreas foram submetidas ao parto vaginal com maior frequência.

Observou-se associação entre antecedentes de partos cesáreos com a utilização de métodos nãofarmacológicos de alívio da dor $(p<0,001)$ e com o oferecimento de líquidos via oral $(p<0,001)$. Parturientes sem história de cesárea anterior foram as que receberam, com maior frequência (49\%), entre dois a quatro métodos não-farmacológicos de alívio da dor e líquidos via oral no trabalho de parto $(60,8 \%)$. As multíparas com antecedentes de partos vaginais (quatro a cinco partos) também receberam, 
com maior frequência, líquidos via oral $(67,7 \%)$. Observou-se, nos hospitais, associação entre receber líquidos via oral e a ocorrência do parto vaginal $(p<0,001)$.

Registrou-se que, dentre os motivos para indicação dos partos cirúrgicos, o principal foi a iteratividade $(26,7 \%)$, o que já era esperado, pois $24 \%$ das mulheres tinham antecedentes de duas cesáreas ou mais.

Outro dado relevante refere-se à alta taxa de cesárea $(48 \%)$ registrada no grupo das primíparas (227 mulheres - 39,9\% da amostra).

\section{Discussão}

$\mathrm{Na}$ média geral de escores, os dois hospitais obtiveram classificação regular e, portanto, a assistência hospitalar ao parto no SUS em Maringá, para o período do estudo, foi considerada regular. Como a alta taxa de cesárea traduz o uso abusivo de tecnologia para o nascimento, infere-se que o resultado da assistência foi insatisfatório.

A média de escore da qualidade, semelhante nos dois hospitais, foi concordante com o obtido por Boaretto, 18 que avaliou a qualidade dos serviços de assistência ao parto no SUS em maternidades do município do Rio de Janeiro, Brasil. A autora observou desempenho semelhante, sem variações importantes, na implementação da política de humanização entre instituições de diferentes naturezas jurídicas (maternidades públicas ou vinculadas ao SUS), evidenciando uma prática de atenção mais ou menos homogênea entre as mesmas.

Entre os sete indicadores, a presença do acompanhante no trabalho de parto (indicador 2) foi o único que apresentou associação com a procedência da parturiente, ou seja, as mulheres procedentes de outros municípios chegavam, frequentemente, sozinhas ao hospital. A baixa escolaridade, o maior número de filhos e as precárias condições socioeconômicas destas mulheres podem justificar o menor suporte social e a ausência do acompanhante. A influência de fatores individuais podem interferir na qualidade da atenção ao parto. Estudo realizado no município de Maringá apontou que ter 35 anos de idade ou mais, ser multigesta, ter baixa escolaridade e antecedentes de cesariana constituíram possíveis fatores predisponentes a iniqüidades na assistência ao parto. 19

Observou-se diferencial no tempo de permanência do acompanhante entre os hospitais analisados. No hospital 1, os acompanhantes permaneceram por mais tempo durante o trabalho de parto, indicando que esta prática mostra-se mais consolidada que na outra instituição. Registrou-se que os acompanhantes das parturientes procedentes de outros municípios permaneceram por menos tempo no trabalho de parto. Achados semelhantes foram encontrados em outros estudos realizados em maternidades públicas brasileiras, o que denota que a prática não foi efetivamente implantada na maioria das maternidades do país. ${ }^{18,20}$

O uso restrito dos métodos não farmacológicos de alívio da dor (indicador 3) e a prática prejudicial de restrição de líquidos durante o trabalho de parto (indicador 4) constituem práticas observadas por outros autores na assistência ao trabalho de parto. 18

A prática da promoção do contato pele-a-pele entre mãe e filho em sala de parto (indicador 7) registrado nesta pesquisa foi superior ao encontrado na literatura brasileira. Corrêa21 observou a ocorrência desta prática em $58 \%$ dos nascimentos obtidos em dois hospitais que receberam o certificado de Hospital Amigo da Criança no município de Santos, Estado de São Paulo, Brasil.

Quando observado o tempo de contato entre mãe e filho, registrou-se que apenas 40 (7\%) crianças tiveram contato pele-a-pele que perdurou por trinta minutos ou mais. Ao considerar-se que o hospital 1 adquiriu o título de Hospital Amigo da Criança em 2003, esperavam-se resultados melhores nas práticas referentes ao estímulo do aleitamento materno.

A associação entre o tipo de parto e qualidade da atenção demonstra que as mulheres submetidas ao parto vaginal receberam atenção mais qualificada quando comparado às que foram submetidas ao parto cesáreo. Sendo assim, a pesquisa indica o que a $\mathrm{WHO}^{9}$ ressalta: o atendimento humanizado qualifica a assistência, contribui para que a evolução do parto ocorra de maneira fi-siológica e favorável, o que culmina com a evolução para o parto vaginal.

Em relação ao uso de tecnologia apropriada para assistência ao parto e nascimento, a $\mathrm{WHO}^{9}$ classifica a cesariana na categoria $\mathrm{D}$, ou seja, é considerada "prática frequentemente utilizada de modo inadequado". Esta assertiva foi confirmada no estudo, pois a taxa de cesarianas encontrada nos dois hospitais constitui o triplo da recomendada pela WHO (15\%) para cesarianas realizadas por indicações estritamente médicas ${ }^{10}$ e distante da taxa de $35 \%$ estabelecida para o Estado do Paraná. ${ }^{17}$ As taxas abusivas expressam uma prática intervencionista arraigada nos serviços de saúde do município, semelhantes às registradas nos últimos anos e concordantes com outros estudos realizados, que assinalaram a inadequação na atenção e a existência de assistência insatisfatória ao parto no município.22,23

A associação entre a hora de ocorrência do parto 
e o tipo de parto, observada nos dois hospitais, também foi relatada no estudo de D'Orsi et al. ${ }^{24} \mathrm{Em}$ pesquisa realizada em hospital público e conveniado ao SUS no Rio de Janeiro os autores registraram que a ocorrência do parto no horário diurno do plantão (entre 9:00 e 15:00 h) e início da noite associou-se à maior chance de realização de cesárea, quando comparados ao período da madrugada. Em relação a este fato, concorda-se com Dias e Domingues, 25 ao explicar que, em princípio, não existe nenhuma justificativa técnica para esta situação. Os autores sugerem que a conveniência da equipe de plantão seja um dos fatores que influencia a escolha pelo parto cesáreo.

Portanto, a existência de associação entre hora de ocorrência do parto e tipo de parto; a influência da história obstétrica na escolha do tipo de parto; e a associação entre antecedentes de partos cesáreos com a utilização de métodos não-farmacológicos de alívio da dor e com o oferecimento de líquidos via oral indicam que a prática dos obstetras pode ser considerada como um dos fatores que explicam a alta taxa de cesárea nos dois hospitais. A alta taxa de cesárea no grupo das primíparas reforça a suposição de que o tipo de parto estava definido previamente à sua evolução.

Estas informações são concordantes com as conclusões obtidas no estudo de D'Orsi et al.,24 de que a elevada proporção de cesárea no Brasil constitui reflexo de três fatores: características das mulheres, práticas dos obstetras e forma de organização da prática obstétrica, da qual a afirmação, preconizada por Cragin26 em 1916, "uma vez cesárea, sempre cesárea”, ainda constitui regra.

A experiência de implantação do parto humanizado em alguns serviços de saúde no Brasil tem se mostrado como um processo lento e, por vezes, difícil. Intervenções múltiplas e consistentes são fundamentais para obtenção do sucesso, tais como: melhoria da qualidade no pré-natal, preparo e incentivo das gestantes para o parto vaginal, difusão de protocolos clínicos e programáticos atualizados, manutenção do debate sobre a qualidade da assistência ao parto e nascimento nas instituições de saúde e nos foros profissionais, investimento finan-

\section{Referências}

1. Laurenti R, Mello-Jorge MHP, Gotlieb SLD. Reflexões sobre a mensuração da mortalidade materna. Cad Saúde Pública. 2000; 16: 23-30

2. Brasil. Ministério da Saúde. Rede Interagencial de Informações para a Saúde (RIPSA). Razão de mortalidade materna, segundo região e UF. Brasil, 2000-2007. [online] 2009. [acesso 24 mai 2011]. Disponível em http://tabnet.datasus.gov.br/cgi/idb2009/ C03b.htm ceiro nos hospitais, oficinas educacionais interativas, capacitação da equipe multiprofissional, entre outros.

Cabe ressaltar que a humanização no cuidado somente começa quando a equipe multiprofissional é capaz de detectar, sentir e interagir com as pacientes e familiares e, principalmente, quando estes podem participar das decisões a respeito dos procedimentos invasivos ou não que deverão ser submetidos, o que inclui a opção pelo parto vaginal.

Uma estratégia que poderia ser adotada para reduzir as taxas de cesárea refere-se à inclusão da enfermeira obstetra na assistência ao parto de baixo risco, a exemplo da Holanda, que apresenta um modelo assistencial ao parto e nascimento que é referência devido as suas baixas taxas de mortalidade materno-infantil. Nesse país, a assistência ao parto e nascimento de baixo risco fundamenta-se na atenção prestada por enfermeiras obstétricas e por parteiras especializadas, cuja formação está voltada para o suporte emocional e o atendimento da mulher e do recém-nascido, sem interferir no processo fisiológico do parto, permitindo à mãe vivenciar esse momento de forma prazerosa e segura. 27

Neste sentido, desde o ano de 1998, o Ministério da Saúde do Brasil tem incentivado a capacitação de enfermeiros na assistência no trabalho de parto e parto mediante o financiamento de cursos de especialização em obstetrícia, bem como a implantação dos centros de parto normal e humanizado. 28

Considerando o grande desafio de reduzir as taxas de cesariana no Brasil, espera-se que este estudo contribua para o reconhecimento de seus determinantes e na construção de políticas públicas e institucionais que garantam atendimento integral, humanizado e de qualidade às mulheres no ciclo gravídico puerperal.

\section{Agradecimentos}

Ao Fundo de Apoio ao Ensino, Pesquisa e Extensão da Universidade Estadual de Campinas (Faepex/Unicamp), pelo apoio financeiro por meio do auxílio linha-pesquisa e auxílio-ponte.

3. Brasil. Ministério da Saúde. Secretaria de Políticas de Saúde. Área Técnica da Saúde da Mulher. Parto, aborto e puerpério: assistência humanizada à mulher. Brasília, DF; 2001.

4. Brasil. Ministério da Saúde. Agência Nacional de Saúde Suplementar. Parto normal versus cesariana: o papel do Estado e das agências reguladoras. Brasília, DF; 2006. 
5. Priszkulnik G, Carrera MA. Parto humanizado: influências no segmento saúde. Mundo Saúde. 2009; 33: 80-8.

6. Stefanini MLR. Editorial. Boletim do Instituto de Saúde. São Paulo: Instituto de Saúde; 2003. 30 (3).

7. Benevides R, Passos E. Humanização na saúde: um novo modismo? Interface 2005; 9 (17): 389-94.

8. Diniz CSG. Humanização da assistência ao parto no Brasil: os muitos sentidos de um movimento. Ciênc Saúde Coletiva. 2005; 10: 627-37.

9. WHO (World Health Organization). Maternal and Newborn Health. Safe Motherhood Unit. Family and Reproductive Health. Care in normal birth: a pratical guide. Genebra; 1996.

10. WHO (World Health Organization). Appropriate technology for birth. Lancet. 1985; 8452: 436-37.

11. Brasil. Ministério da Saúde. Programa de humanização no pré-natal e nascimento: informações para gestores e técnicos. Brasília: Secretaria de Políticas de Saúde. Área Técnica da Saúde da Mulher; 2000. 33p.

12. Brasil. Ministério da Saúde. Sistema Nacional de Informação de Nascidos Vivos. Nascidos vivos ocorridos em Maringá-Paraná por tipo de parto. Maringá-Paraná, 2003, 2004, 2005 [online] 2006 [acesso 26 mar 2006]. Disponível em: www.datasus.gov.br/catalogo/sinasc.htm

13. Worthen BR, Sanders JR, Fitzpatrick JL. Avaliação de programas: concepções e práticas. São Paulo: Editora Gente; 2004. 730p.

14. Hodnett ED, Gates S, Hofmeyr GJ, Sakala C. Continuou support for women during childbirth (Cochrane Review) [online]. 2011. [acesso 24 mai 2011]. Disponível em: http://www2.cochrane.org/reviews/en/ab003766.html

15. Enkin M, Keirse MJNC, Neilson J, Duley CCLD, Hodnett $\mathrm{E}$, Hofmeyr J. A guide to effective care in pregnancy and childbirth. [online]. 2000. [acesso 24 mai 2011]. Disponível em: http://www.childbirthconnection.org/article.asp? ClickedLink $=194 \& \mathrm{ck}=10218 \&$ area $=2$

16. Simkin PP, O'Hara M. Nonpharmacologic relief of pain during labor: systematic reviews of five methods.Am J Obstet Gynecol. 2002; 186: 131-59.

17. Brasil. Ministério da Saúde. Departamento Nacional de Auditoria do SUS. Coordenação de Sistemas de Informação. Sistema Único de Saúde-Legislação Federal. Portaria MS/GM n ${ }^{\circ}$ 466, de 14 de junho de 2000. Estabelece um modelo de "Pacto para Redução das Taxas de Cesarianas" a ser firmado entre os Estados, Distrito Federal e o Ministério da Saúde. Brasília, DF; 2000.
18. Boaretto MC. Avaliação da política de humanização do parto e nascimento no município do Rio de Janeiro [Dissertação]. Rio de Janeiro: Escola Nacional de Saúde Pública da Fundação Oswaldo Cruz; 2003.

19. Nagahama EEI, Santiago SM. Humanização e equidade na atenção ao parto em município da região Sul do Brasil. Acta Paul Enf. 2008; 21: 609-15.

20. Domingues RMSM. Acompanhantes familiares na assistência ao parto normal: a experiência da Maternidade Leila Diniz [Dissertação]. Rio de Janeiro: Escola Nacional de Saúde Pública da Fundação Oswaldo Cruz; 2002.

21. Corrêa MAP. Relação mãe-bebê no pós-parto: uma contribuição para a Iniciativa Hospital Amigo da Criança [Dissertação]. São Paulo: Programa de Pós-graduação em Ciências, Instituto de Pesquisa da Secretaria de Estado de Saúde de São Paulo; 2004.

22. Silva RLDT. Cesáreas: frequência e consequências maternas e perinatais - Maringá-PR, 1995 [Dissertação] Londrina: Universidade Estadual de Londrina; 1997.

23. Serafim D. Mortalidade neonatal em Maringá-Paraná, 1997-2000 [Tese]. São Paulo: Universidade de São Paulo;2002.

24. D'Orsi E, Chor D, Giffin K, Ângulo-Tuesta A, Barbosa GP, Gama AS. Factors associated with cesarean sections in a public hospital in Rio de Janeiro, Brazil. Cad Saúde Pública. 2006; 22 (10): 2067-78

25. Dias MAB, Domingues RMSM. Desafios na implantação de uma política de humanização da assistência hospitalar ao parto. Ciênc Saúde Coletiva. 2005; 10: 669-705.

26. Cragin EB. Conservatism in obstetrics. NY Med J. 1916; 104: 1-3.

27. Brasil. Ministério da Saúde. Portaria MS/GM n 985 , de 05 de agosto de 1999. Cria o Centro de Parto Normal e estabelece normas e critérios de inclusão no SUS. Brasília, DF; 1999.

28. Brasil. Ministério da Saúde. Resolução-RDC n 36, de 03 de junho de 2008. Dispõe sobre regulamento técnico para funcionamento dos serviços de atenção obstétrica e neonatal. Diário Oficial da União [DOU]. Brasília, 04 de junho de 2008 .

Recebido em 27 de janeiro de 2011

Versão final apresentada em 13 de outubro de 2011

Aprovado em 31 de outubro de 2011 